\title{
Melaza de melón de descarte, una alternativa sustentable, para una producción de
}

\section{melón responsable}

\author{
Discard melon molasses, a sustainable alternative, to response melon production \\ Melaço de melão de refugo, uma alternativa sustentável, para uma produção de melão responsável
}

\author{
Salvador González Chacón \\ ORCID: https://orcid.org/0000-0002-6401-7384 \\ Universidade Federal Rural do Semiárido, Brasil \\ E-mail: mrguinox@gmail.com \\ Patrícia de Oliveira Lima \\ ORCID: https://orcid.org/ 0000-0002-1887-3446 \\ Universidade Federal Rural do Semiárido, Brasil \\ E-mail: pattlima@ufersa.edu.br \\ Lerner Arévalo Pinedo \\ ORCID: https://orcid.org/0000-0001-8119-8626 \\ Universidade Federal Rural do Semiárido, Brasil \\ E-mail: lernerpinedo@gmail.com \\ Rebeca Bertin Afonso \\ ORCID: https://orcid.org/0000-0002-8547-6643 \\ Universidade Federal Rural do Semiárido, Brasil \\ E-mail: rebeca.afonso@alunos.ufersa.edu.br \\ Salenilda Soares Firmino \\ ORCID: https://orcid.org/0000-0003-2596-7210 \\ Universidade Federal Rural do Semiárido, Brasil \\ E-mail: salenildafirmino@hotmail.com \\ Ana Michel Garcia Varela \\ ORCID: https://orcid.org/0000-0002-8667-8554 \\ Universidade Federal Rural do Semiárido, Brasil \\ E-mail: garciavarela126@gmail.com \\ Alejandro Jose Lopez Gonzalez \\ ORCID: https://orcid.org/0000-0002-6198-6585 \\ Universidad Nacional Experimental del Tachira, Venezuela \\ E-mail: Lopezob85@gmail.com
}

\section{Resumen}

El nordeste de Brasil se caracteriza por ser una región importante para la producción de melón a nivel mundial. Actualmente a los estados del Brasil Ceará (CE) y Rio Grande del Norte (RN), se les atribuye la producción de alrededor del $90 \%$ del melón producido en el país, tanto para la exportación como para el consumo interno, lo que abre un abanico de oportunidades para la utilización del melón de descarte como materia prima principal en productos alimenticios alternativos y emprendimientos. La siguiente investigación propone la elaboración y caracterización de melaza de melón a partir de melón de descarte de tres distintas variedades producidas en rio grande del norte, Pele de sapo, Cucumis melo 'Santa Claus', Amarillo Cucumis melo L. (Inodorus Group) 'Canary' y verde, Cucumis melo L. (Inodorus Group) 'Honey $D e w^{\prime}$. Los análisis físico-químicos fueron realizados en el laboratorio de tecnología de alimentos de la Universidad Federal Rural del Semiárido. Dichos análisis fueron: Sólidos solubles, SS (oBrix), pH, Acidez titulable, (ATT) (\%, ácido cítrico), Conductividad eléctrica, $(\mathrm{mS} / \mathrm{cm})$, Azúcares totales $(\%, \mathrm{~g} / 100 \mathrm{~g})$, Fenoles totales $(\mathrm{EAG}, \mathrm{mg} / 100 \mathrm{~g})$ $\mathrm{EAG}=$ Ácido gálico equivalente, Actividad antioxidante (IC50, $\mathrm{mg} / \mathrm{mL}$ ). Esta investigación promueve y contribuye a alcanzar los objetivos de desarrollo sostenible (ODS) propuestos por la ONU en su agenta 2030, específicamente el objetivo $\mathrm{N}^{\circ} 12$, "Producción y consumo responsable".
\end{abstract}

Palabras clave: Melón de descarte; Emprendimiento; Melaza.

\begin{abstract}
Northeast Brazil is characterized as an important region for melon production worldwide. Currently, the states of Brazil Ceará $(\mathrm{CE})$ and Rio Grande do Norte $(\mathrm{RN})$ are attributed the production of around $90 \%$ of the melon produced in the country, both for export and for domestic consumption, which opens a range of opportunities for the use of discarded melon as the main raw material in alternative food products and ventures. The following research proposes the elaboration and characterization of melon molasses from discarded melon of three different varieties produced in Rio Grande do Norte, Pele de sapo, Cucumis melo 'Santa Claus', Yellow Cucumis melo L. (Inodorus Group)' Canary 'and
\end{abstract}


green, Cucumis melo L. (Inodorus Group)' Honey Dew '. The physical-chemical analyzes were carried out in the food technology laboratory of the Semi-arid Rural Federal University. These analyzes were: Soluble solids, SS (oBrix), pH, Titratable acidity, (ATT) (\%, citric acid), Electrical conductivity, $(\mathrm{mS} / \mathrm{cm})$, Total sugars $(\%, \mathrm{~g} / 100 \mathrm{~g})$, Total phenols $(\mathrm{EAG}, \mathrm{mg} / \mathrm{100 \textrm {g }}) \mathrm{EAG}=$ Gallic acid equivalent, Antioxidant activity (IC50, mg / mL). This research promotes and contributes to achieving the sustainable development goals (SDG) proposed by the UN in its 2030 agenda, specifically goal No. 12, "Responsible consumption and production".

Keywords: Discard melon; Entrepreneurship; Molasses.

\section{Resume}

O Nordeste do Brasil é caracterizado como uma importante região para a produção de melão em todo o mundo. Atualmente, aos estados do Brasil Ceará (CE) e Rio Grande do Norte (RN) é atribuída a produção de cerca de $90 \%$ do melão produzido no país, tanto para exportação quanto para consumo interno, o que abre um leque de oportunidades para a utilização do melão de refugo como principal matéria-prima em produtos alimentícios alternativos e empreendedorismo. A pesquisa a seguir propõe a elaboração e caracterização de melaço de melão de refugo de três diferentes variedades produzidas no Rio Grande do Norte, Pele de sapo, Cucumis melo 'Santa claus', Amarelo Cucumis melo L. (Grupo Inodorus) 'Canário' e verde, Cucumis melo L. (Grupo Inodorus) 'Honey Dew'. As análises físicoquímicas foram realizadas no Laboratório de Tecnologia de Alimentos da Universidade Federal Rural do Semiárido. Essas análises foram: sólidos solúveis, SS (oBrix), pH, acidez titulável, (ATT) (\%, ácido cítrico), condutividade elétrica, $\mathrm{CE}(\mathrm{mS} / \mathrm{cm})$, açúcares totais AST $(\%, \mathrm{~g} / 100 \mathrm{~g})$, fenóis totais $(\mathrm{EAG}, \mathrm{mg} / 100 \mathrm{~g})$ EAG = equivalente de ácido gálico, atividade antioxidante AT (IC50, mg / ml). Esta pesquisa promove e contribui para o alcance dos objetivos de desenvolvimento sustentável (ODS) propostos pela ONU em sua agenda 2030, especificamente o objetivo $\mathrm{n}^{\circ} 12$, "Consumo e produção responsáveis".

Palavras-chave: Melão de refugo; Empreendedorismo; Melaço.

\section{Introducción}

Según Araujo (2018) La preocupación por el medio ambiente despierta el interés por evitar el desperdicio en la cadena de producción de alimentos, que es responsable de la generación de cantidad considerable de residuos. Unido a esto, el uso de residuos de frutas, puede agregar valor nutricional a nuevos productos. El volumen de exportaciones ha aumentado en 2019 , tanto para frutas como para hortalizas. En el caso del melón con 404.511 toneladas y 287,5 millones de euros (ICEX. 2020). Concierne al melón se comercializa la producción en su totalidad en forma de fruta fresca, que es la forma de consumo más común. Los mercados nacionales, regionales y locales consumen el 70\% de la producción y el mercado exterior el $20 \%$, el $10 \%$ restante es desperdicio de fruta. (Correia de lima et, al. 2012).

La melaza proviene de la cristalización del jugo concentrado. Una parte es empleada para la alimentación animal y otra se utiliza en la elaboración de alcohol carburante para la exportación, para la producción de lácteos y numerosos productos biotecnológicos. Los principales componentes de la melaza son el agua y los carbohidratos. (Vega. J. et, al. 2007). La melaza producto residual de la industria azucarera empleada en la alimentación animal, se diferencia de la melaza que es empleada como materia prima en la producción de azúcar, un ejemplo es la melaza de remolacha que no es apta para el consumo humano pues es amarga, sin embargo, se utiliza en la alimentación de ganado vacuno. Entre los usos de estos jarabes pueden reemplazar el azúcar, lo que los hace productos adecuados para los suministros para diabéticos; también de la melaza se pueden obtener diversos tipos de alcoholes mediante fermentación. (Vásquez 2017).

Según Cárdenas (2018) la melaza en la concentración del 20\% es apta para la producción de las bacterias ácido lácticas; además, las condiciones a las cuales fueron sometidos los biorreactores permitieron que el microorganismo se adaptara, de esta forma tuviera una producción de biomasa y fermentación. Los resultados obtenidos, permitieron definir que la melaza es un medio alternativo, de bajo costo para la producción de biomasa a partir de bacterias ácido lácticas. La melaza se emplea como alimento de ganado ovino, vacuno, cerdos y aves, debido a que contienen hidratos de carbono, materia inorgánica, oligoelementos, y relativamente pocas proteínas, por lo cual es mejor sintetizada como alimento de rumiantes, estos descomponen las células bacterianas como fuente de nitrógeno en la síntesis de proteínas bacterianas. (Vásquez 2017).

El desperdicio de alimentos tiene un gran impacto en la sociedad y el medio ambiente. Pérdidas se observan a lo largo 
de toda la cadena productiva, lo que establece la necesidad de uso completo de los alimentos, así como la posibilidad de mantenimiento del consumo habitual con el uso de residuos para nuevos productos alimenticios. (Curado. R. 2012). Residuos vegetales que normalmente se pasan por alto, demuestran un gran potencial para ser reutilizados como materia prima en el desarrollo de nuevos productos. (Gouveia 2014). La evolución tecnológica y una mayor conciencia de responsabilidad social, ambiental y sostenibilidad de la producción, buscan cada vez más alternativas para reemplazar las fuentes tradicionales de energía y proteína (De Lima 2020). El uso de subproductos vegetales y frutales como materia prima, demuestra ser una excelente opción para el enriquecimiento nutricional de nuevos productos. Los artículos existentes en la literatura demuestran que estos residuos son responsables del aumento de la cantidad de fibras, vitaminas y minerales, mejorando el funcionamiento del organismo y actuando en la prevención de diversas patologías. (Gouveia 2014). Dada a la gran importancia que tiene la producción y consumo responsables, junto a la oportunidad que representa el melón de descarte. Esta investigación propone fabricar melaza a partir del jugo de tres variedades distintas de melón de descarte y realizar su caracterización físico-química y su potencial antioxidante para utilización en la industria de la nutrición humana o animal.

\section{Metodología}

\subsection{Metodología aplicada en la investigación}

El tipo de investigación a realizar es descriptivo, según Arias (2006) la investigación descriptiva consiste en la caracterización de un hecho, fenómeno, individuo o grupo, con el fin de establecer su estructura o comportamiento. Así mismo Tamayo, (2002) la define como la descripción, registro, análisis e interpretación de la naturaleza actual, y la composición o proceso de los fenómenos. También el estudio se puede clasificar como una investigación de tipo experimental ya que se van a evaluar el comportamiento de variables bajo ciertas condiciones establecidas. Tamayo, (2002) afirma que la investigación experimental se presenta mediante la manipulación de una variable experimental no comprobada, en condiciones rigurosamente controladas, con el fin de describir de qué modo o por qué causa se produce una situación o acontecimiento particular. Los frutos de melón fueron obtenidos por donación de productores de melón ubicados en Mossoró, Rio Grande del Norte, Brasil. Las variedades utilizadas para la fabricación de la melaza de melón fueron: Pele de sapo, Cucumis melo 'Santa Claus', Amarillo Cucumis melo L. (Inodorus Group) 'Canary' y verde, Cucumis melo L. (Inodorus Group) 'Honey Dew'.

\subsection{Técnica de preparación de la melaza de melón}

La preparación de la melaza se llevó a cabo en las instalaciones de la Universidad Federal Rural del Semiárido, los melones maduros fueron lavados, posterior mente fue extraída la cascara para poder licuarlos con más facilidad, se procedió a licuar la fruta incluyendo la semilla, hasta obtener dos litros exactos de cada variedad y dos litros de la mezcla de los jugos de las tres variedades en conjunto. Posteriormente se realizó el filtrado del jugo hasta obtener jugo concentrado sin impurezas. El jugo concentrado obtenido fue colocado en cuatro recipientes para posteriormente realizar la cocción con la misma cantidad de fuego. Los fogones fueron apagados después de transcurrida una hora con veintiocho minutos, cuando el jugo fue reducido por el calor y dio punto de melaza. Finalmente se dejaron reposar las muestras obtenidas y se colocaron en envases plásticos de $100 \mathrm{ml}$ totalmente esterilizados. Se formularon cuatro tratamientos diferentes por cada parámetro analizado, cada tratamiento figura como cada una de las melazas fabricadas a partir de distintas variedades de melón y la mezcla de los tres, denominadas, Amarillo A= Cucumis melo L. (Inodorus Group) 'Canary'; Verde V= Cucumis melo L. (Inodorus Group) 'Honey Dew; Pele de sapo $\mathrm{P}=$ Cucumis melo 'Santa Claus' y finalmente Mezcla= la mezcla de los tres en partes iguales. 


\subsection{Análisis físico-químicos realizados}

Los análisis físico-químicos de las cuatro muestras de melaza obtenida fueron realizados en la Universidad Federal Rural do Semiárido - UFERSA, en el Laboratorio de Tecnología de Alimentos.

\subsection{Descripción de los análisis realizados}

\subsection{1 pH}

El pH fue determinado mediante potenciómetro, según la técnica (AOAC, 1992).

\subsubsection{Conductividad eléctrica}

La conductividad eléctrica se analizó en microsiemens/centímetro $(\mu \mathrm{S} / \mathrm{cm})$ siguiendo la metodología empleada por la (AOAC, 1992)

\subsubsection{Los sólidos solubles (SS)}

Se determinaron utilizando el refractómetro digital modelo PR-100 Palette (Attago Co. Ltd., Japón), con corrección automática de temperatura y lectura en el rango de 0 a 32\%, los resultados expresados como porcentaje (\%) (AOAC, 1992).

\subsubsection{La acidez total titulable (ATT)}

Se determinó mediante titulación (Instituto Adolf Lutz, 2008) a partir de $5 \mathrm{~g}$ de muestra, a los que se agregaron $40 \mathrm{ml}$ de agua destilada. Luego, se realizó la titulación con una solución estandarizada de $\mathrm{NaOH} 0.1 \mathrm{~N}$, determinándose el punto final de la titulación con la ayuda del potenciómetro digital hasta alcanzar un $\mathrm{pH}$ de 8.1. Los resultados se expresan como \% de ácido cítrico.

\subsubsection{Los azúcares solubles totales (AST)}

Se determinaron mediante el método del reactivo de antrona, según Yemn y Willis (1954). Se utilizó 0,1 gramos de muestra diluida con agua destilada hasta $200 \mathrm{ml}$. A continuación, se retiraron alícuotas de $100 \mu 1$ y se añadieron $2 \mathrm{ml}$ de reactivo de antrona ( $2 \mathrm{~g} / \mathrm{L}$ en H2SO 4 concentrado) a un tubo de ensayo seguido de calentamiento durante 15 minutos. Las absorbancias obtenidas se colocaron en un espectrofotómetro con una longitud de onda de $620 \mathrm{~nm}$, utilizando glucosa como estándar y los resultados expresados como porcentaje (\%).

\subsubsection{Actividad antioxidante (AT)}

Se midió de acuerdo con el método de reducción de radicales libres 2,2-difenil-1-pricilhidrozil (DPPH), descrito por (Velázquez. et al. 2003), con algunas modificaciones. Con la presencia de un reactivo antioxidante, el color púrpura del DPPH disminuye, por lo que se puede leer mediante espectrofotometría debido al cambio de absorbancia. Una alícuota de $1 \mathrm{ml}$ de cada dilución (2-10 mg/ml) de muestra de melaza de melón fue adicionado $1,5 \mathrm{ml}$ de DPPH (0,029 mg/ml) y luego de ser agitados, los tubos fueron dejados en reposo protegidos de la luz por 15 minutos. Las lecturas de absorbancia se realizaron utilizando el espectrofotómetro Gehaka modelo UV-340G a $517 \mathrm{~nm}$ utilizando el control de $1 \mathrm{ml}$ de metanol y DPPH. Así, la actividad antioxidante de los extractos se obtuvo considerando el porcentaje de inhibición del radical DPPH, calculado según la siguiente ecuación.

$\%$ Inhibición $=[($ Control absoluto - Muestra absoluta $) /$ Control absoluto $] \times 100$ 
Se trazaron curvas de \% de inhibición frente a concentración para cada extracto con el fin de calcular el valor de IC50. Los parámetros de regresión lineal se planificaron para cada curva en el software Origin 7.0.

\subsubsection{Fenoles totales}

Se determinaron de acuerdo con la metodología de Meda et al. (2005), utilizando el reactivo de Folin-Ciocalteau. De muestras de melaza de melón ( $50 \mathrm{~g} / \mathrm{L})$, se retiraron alícuotas de $0.5 \mathrm{~mL}$, se adicionó $2.5 \mathrm{ml}$ del reactivo Folin-Ciocalteau (0.2N) en un tubo de ensayo, luego de 5 minutos, se colocaron $2 \mathrm{ml}$ de carbonato de sodio $(75 \mathrm{~g} / \mathrm{L})$ después se mantuvo durante 2 horas en la oscuridad. La absorbancia se midió en espectrofotometría Gehaka modelo UV-340G a 760 nm, utilizando metanol blanco. Los resultados obtenidos se extrapolaron en una curva de calibración obtenida con ácido gálico estándar (20 a 200 ppm), y se expresaron en mg de ácido gálico equivalente (EAG) /gr de muestra.

\section{Resultados y discusión}

Las muestras analizadas de las cuatro variedades de melaza preparadas arrojaron un resultado homogéneo en cuanto a lo que concierne a los ${ }^{\circ}$ Brix. La única muestra que vario fue la muestra de melaza preparada con melón amarillo, la cual arrojo como resultado una medida superior a la que el refractómetro utilizado alcanza a leer. La cantidad de Brix ${ }^{\circ}$ obtenida indica la cantidad de solidos solubles presentes en las muestras, por ende, azucares que en el caso de melaza de melón arrojo alrededor del 50 \%. Según (Da silva. F .2010) La Melaza es el jugo de caña de azúcar concentrado por la evaporación del agua hasta que alcance un tenor de sólidos (Brix) entre 65 y 75\%. La temperatura del concentrado, debe ser de aproximadamente $103^{\circ} \mathrm{C}$ cuando alcance $65^{\circ}$ Brix, y $105^{\circ} \mathrm{C}$ a $72^{\circ}$ Brix.

Tabla 1. ${ }^{\circ}$ Brix

\begin{tabular}{|c|c|c|}
\hline Muestra & SS, Brix $^{\circ}$ & $\pm \mathrm{SD}$ \\
\hline Mezcla & 50,2 & 0,2 \\
\hline $\mathrm{P}$ & 50,2 & 0,1 \\
\hline A & $>53$ & - \\
\hline V & 50,5 & 0,1 \\
\hline
\end{tabular}

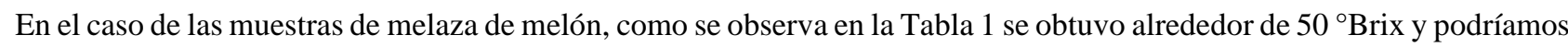
aseverar que corresponde a azucares. Vale la pena resaltar la gran diferencia obtenida al comparar la melaza de melón por ejemplo con la de caña de azúcar, la cual tiene según la mayoría de autores entre 70 y $80{ }^{\circ}$ Brix, en el ámbito de la industria de alimentos para animales esta es una característica deseada, ya que, la melaza actúa como aglomerante. En el caso de ser ofertada directamente a los animales o en ensilaje esta cantidad de ${ }^{\circ}$ Brix es no deseada, ya que, por su alto grado de viscosidad por lo general debe ser diluida en agua. Para la producción de biomasa es importante que la melaza tenga menos de $55^{\circ} \mathrm{Brix}$, de lo contrario debe clarificarse hasta alcanzar el nivel óptimo para utilizarse lo que no ocurriría en el caso de la melaza de melón.

El pH está relacionado con el número comparativo de los iones hidrogenados $(\mathrm{h})^{+} \mathrm{o}$ de los iones de oxhidrilo $\left(\mathrm{OH}^{-}\right)$dentro de una solución. Una solución ácida contiene un número más alto de iones hidrogenados; las soluciones alcalinas tienen un número relativo más alto de iones ${ }^{-}$del $\mathrm{OH}$. En el caso de la medición del pH de las melazas de melón analizadas, todas tienen valores ligeramente ácidos, la menos acida del grupo fue la melaza de melón verde con un pH de 6,22 Tabla 2. Los valores no presentaron diferencia a los valores que comúnmente se atribuyen a melaza fabricada a partir de caña de azúcar, que según varios 
autores se encuentra entre 5,5 y 6,5 y que por ejemplo en la industria de producción de biomasa para fermentación suele ajustarse a 5,0 .

Según Moro (2012) Después de realizar el un estudio para mejorar los procesos de fermentación alcohólica. Recomienda

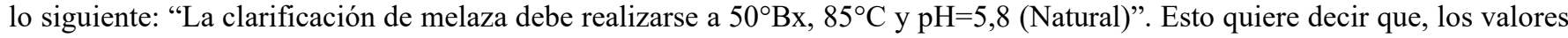
anteriormente discutidos hallados en la melaza de melón se ajustan, a los recomendados para utilizar en la fermentación alcohólica, sin necesidad de realizar ajustes. En cuanto a la producción de ensilaje, se recomienda al incluir melaza a su preparación a un pH ligeramente ácido para favorecer la proliferación de bacterias acido lácticas que usan la melaza como sustrato para su proliferación y brindan una mejor calidad al producto ensilado, aparte de que inhiben el crecimiento de colonias de microorganismos indeseados.

La acidez titulable se realiza en base al acido presente en el melón, en este caso se analizó la cantidad de ácido cítrico presente en las muestras de melaza de melón. Este análisis permite cuantificar la concentración de ácido a partir de una valoración acido-base. Según (Lemus \& Mendoza 2011) Para procesos en la industria alimenticia como por ejemplo para la fabricación de néctares la acidez optima varía de un 0.60 a un $0.70 \%$.

Tabla 2. pH, Acides titulable y conductividad eléctrica de muestras de melaza de melón de descarte.

\begin{tabular}{|c|c|c|c|c|c|c|}
\hline \multirow{3}{*}{$\begin{array}{l}\text { Muestra pH } \\
\text { Mezcla }\end{array}$} & \multirow{2}{*}{\multicolumn{2}{|c|}{$\pm \mathrm{SD}$}} & \multirow{3}{*}{$\begin{array}{c}\text { ATT (\%,ác. cítrico) } \\
\mathbf{0 , 5 1 9}\end{array}$} & \multicolumn{3}{|c|}{ CE } \\
\hline & & & & $\pm \mathrm{SD}$ & $\mathrm{mS} / \mathrm{cm}$ & $\pm \mathrm{SD}$ \\
\hline & 5,85 & 0,2 & & 0,006 & 3,255 & 0,36 \\
\hline $\mathrm{P}$ & 5,81 & 0,15 & 0,413 & 0,001 & 4,460 & 0,29 \\
\hline A & 5,68 & 0,11 & 0,506 & 0,008 & 4,195 & $\mathbf{0 , 3 5}$ \\
\hline V & 6,22 & 0,18 & 0,324 & 0,003 & 3,800 & 0,23 \\
\hline
\end{tabular}

Muestra: Mezcla: Melaza de la mezcla de las tres variedades de melón; P: Melaza de melón Pele de Sapo; A: Melaza de melón Amarillo; V: Melaza de melón Verde; ATT: acides titulable; CE: Conductividad eléctrica.

En el caso de las muestras analizadas de melaza de melón, el \% ATT tuvo diferencia notable entre la melaza obtenida de la mezcla de los tres jugos de melón "Mezcla" comparada con dos variedades la P y la V que fue la que contuvo menos porcentaje de ácido cítrico con $0,324 \%$. El \% de ácido cítrico obtenido en las muestras está por debajo del que podría ser perjudicial para la salud humana por ejemplo en la industria de bebidas y alimentos. Este acido presente naturalmente en las melazas de melón analizadas, es añadido artificialmente en la industria de alimentos como antioxidante, acidulante y saborizante, también es ampliamente usado en medicamentos, esto da una ventaja nutricional a la melaza de melón de descarte.

La evaluación de los azucares solubles totales en las muestras de melaza arrojaron resultados similares tanto en las variedades de melón separadas, como en la mezcla de las tres variedades juntas, la variedad con el mayor porcentaje de azucares solubles totales fue la de melón amarillo con $31,85 \%$ de azucares y la menor la de melón verde con 28,41\% de azucares solubles totales.

Tabla 3. Azucares totales.

\begin{tabular}{lcc} 
Muestras & AST $(\%)$ & \pm SD \\
Mezcla & $\mathbf{2 9 , 6 8}$ & $\mathbf{0 , 8 8 0}$ \\
P & $\mathbf{2 9 , 2 2}$ & $\mathbf{0 , 2 0 3}$ \\
A & $\mathbf{3 1 , 8 5}$ & $\mathbf{0 , 1 1 0}$ \\
V & $\mathbf{2 8 , 4 1}$ & $\mathbf{0 , 1 0 0}$ \\
& & \\
\hline
\end{tabular}


Muestra: Mezcla: Melaza de la mezcla de las tres variedades
de melón; P: Melaza de melón Pele de Sapo; A: Melaza de
melón Amarillo; V: Melaza de melón Verde; AST: azucares totales.

Los resultados que se observan en la Tabla 3, se pueden superponer con el comportamiento de las melazas en el análisis de los ${ }^{\circ}$ Brix, ya que ese índice refleja la cantidad de azucares presentes en cada muestra y en efecto la cantidad de azucares solubles totales y grados brix fue mayor en la variedad de melón A (Vásquez. L. 2017) reporta en su investigación una cantidad de azucares solubles totales de 11,9\% en melaza de sub producto de plátano (Musa abb Simmonds) colocándola por debajo del porcentaje de AST obtenido en las melazas de melón de descarte. Según (FEDNA. 2020) La melaza de caña de azúcar tiene un total de 46\% de AST, en este caso la cantidad de azucares se eleva en comparación a los AST obtenidos en las muestras de melaza de melón analizadas.

La conductividad eléctrica entre las melazas de melón no tuvo una gran varianza, todas obtuvieron valores entre 3 y 4,5 $\mathrm{mS} / \mathrm{cm}$. La melaza obtenida a partir de la mezcla del jugo de las tres variedades de melón, obtuvo el valor más bajo de conductividad eléctrica con $3,2 \mathrm{mS} / \mathrm{cm}$. (Naranjo. W. 2008) En una investigación hecha en ecuador, realizo la caracterización de la miel de caña de dos variedades de caña distintas variedades POJ 28-78 y POJ 27-14 respectivamente, entre los análisis realizados, se evaluó la conductividad eléctrica, el autor al realizar este análisis obtuvo valores que variaron entre $0,15 \mathrm{mS} / \mathrm{cm}$ y $0,69 \mathrm{mS} / \mathrm{cm}$ dependiendo de la variedad de caña estudiada. Comparando este resultado podemos discernir que la melaza de melón posee una concentración de sales y minerales casi seis veces mayor a la miel de caña de azúcar estudiada por el autor citado, lo que le da un atractivo mayor para la industria de alimentos.

En cuanto a la determinación de fenoles totales, los resultados se expresan en mg equivalente de ácido gálico por gramo de muestra, el ácido gálico es utilizado como estándar de calibración para cuantificar fenoles siendo un compuesto fenólico puro. En la Tabla 4 se observa que de las melazas de melón analizadas por el método de Folinciocalteu, la melaza $\mathrm{V}$ obtuvo una mayor concentración de fenoles de 213,33 EAG mg/g seguida por A y P, que tuvieron 182,95 y 172,89 EAG mg/g respectivamente, finalizando con la melaza fabricada con la mezcla del jugo de los tres melones que obtuvo 144,77 EAG mg/g.

Tabla 4. Fenoles.

\begin{tabular}{lcc} 
Amostra & EAG mg/g & \pm SD \\
Mezcla & $\mathbf{1 4 0 , 7 7}$ & $\mathbf{1 , 4 2}$ \\
P & $\mathbf{1 7 2 , 8 9}$ & $\mathbf{0 , 7 0}$ \\
A & $\mathbf{1 8 2 , 9 5}$ & $\mathbf{2 , 3 9}$ \\
V & $\mathbf{2 1 3 , 3 3}$ & $\mathbf{0 , 7 2}$ \\
\hline
\end{tabular}

Muestra: Mezcla: Melaza de la mezcla de las tres variedades de melón; P: Melaza de melón Pele de Sapo; A: Melaza de melón Amarillo; V: Melaza de melón Verde. EAG: equivalente acido gálico.

Las autoras (Amaya \& Portillo 2013) determinaron la cantidad de fenoles en azúcar moreno, azúcar blanco y melaza de caña de azúcar, en su experiencia las autoras señalan haber obtenido en promedio 828,86 EAG mg/g en la melaza de caña, 11,77 EAG mg/gr en el azúcar moreno y 4,14 EAG mg/gr en el azúcar blanco, comparando con estos resultados todas las melazas fabricadas con las distintas variedades de melón de descarte, obtuvieron una cantidad mayor de fenoles que el azúcar blanco y el azúcar moreno, sin embargo resultaron con menor cantidad de fenoles que la melaza de caña. En cuanto a las melazas analizadas en nuestra investigación, la melaza de melón verde obtuvo la mayor concentración de fenoles y por ende un mayor potencial antioxidante. 
La concentración apropiada para inhibir la mitad de la inhibición máxima (IC50) es una medida de la efectividad de un compuesto para inhibir la función biológica o bioquímica, que vincula inversamente el porcentaje de actividad con la concentración de la sustancia probada. Por tanto, cuanto menor sea el valor de IC50, mayor será la capacidad antioxidante de la sustancia.

Tabla 5. Índice IC50.

\begin{tabular}{lcc} 
Muestra & IC50 $(\mathrm{mg} / \mathrm{mL})$ & \pm SD \\
Mezcla & $\mathbf{1 2 , 5 3}$ & $\mathbf{0 , 2 8}$ \\
P & $\mathbf{8 , 0 0}$ & $\mathbf{0 , 1 8}$ \\
A & $\mathbf{7 , 8 2}$ & $\mathbf{0 , 0 5}$ \\
V & $\mathbf{4 , 8 6}$ & $\mathbf{0 , 1 4}$ \\
\hline
\end{tabular}

Muestra: Mezcla: Melaza de la mezcla de las tres variedades de melón; P: Melaza de melón Pele de Sapo; A: Melaza de melón Amarillo; V: Melaza de melón Verde.

En cuanto a la concentración de IC50 en la Tabla $\mathrm{N}^{\circ}$ 5, la actividad de la capacidad de captación de radicales libres DPPH•

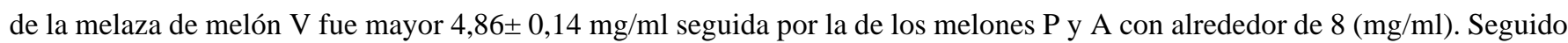
por la mezcla del jugo de los tres melones que obtuvo una captación de radicales libres menor de 12,53 (mg/ml). Los valores de IC50 fueron bastante variables entre las muestras analizadas y demuestran que la melaza de melón de descarte tiene actividad antioxidante y al realizar una melaza de melón de las tres variables, su potencial antioxidante disminuye.

\section{Consideraciones Finales}

Las melazas obtenidas a partir de melones de descarte de distintas variedades de la fruta producida en el nordeste de Brasil, arrojaron resultados favorables. Las tres variedades de melón tuvieron comportamiento similar, destacando un $\mathrm{pH}$ ligeramente acido y una cantidad de grados Brix que potencian su utilización en la fabricación de productos obtenidos vía fermentación como alcoholes y ensilajes. El \% de ácido cítrico obtenido en las muestras está por debajo del que podría ser perjudicial para la salud humana por ejemplo en la industria de bebidas y alimentos, la conductividad eléctrica obtenida destaco por encima de la obtenida en melazas de caña, alcanzando por ende una mayor cantidad de sales y minerales en las melazas de melón. En cuanto al potencial antioxidante las melazas de melón tuvieron un comportamiento favorable y manifestaron tener potencial antioxidante, pudiéndose comparar su potencial antiradicalario por ejemplo con productos antioxidantes naturales como miel de abeja, destacando entre todas las variedades, la melaza de melón V sobre las demás melazas analizadas. En general la melaza de melón de descarte de las variedades Pele de sapo, Cucumis melo 'Santa Claus', Amarillo Cucumis melo L. (Inodorus Group) 'Canary' y verde, Cucumis melo L. (Inodorus Group) 'Honey Dew' y la mezcla de las tres variedades, destacaron sobre productos similares y suma sustentabilidad a la cadena de producción de melón al utilizar melones descartados para crear un sub producto útil para distintos fines como la industria de alimentos animal y humana, la fabricación del alcoholes entre otros, se recomienda realizar análisis bromatológicos, testear en animales características sensoriales y estudios de fermentación in vitro para la producción de alcoholes.

\section{Referencias}

Arias, F. (2006) El proyecto de la investigación; introducción a la metodología científica. EPISTEME.. 
Cardenas, L.M., Gómez, J.A., Arenas, M., \& Serna Jiménez, J. (2018). Evaluación de melaza como medio de cultivo para la producción de bacterias ácidolácticas. UG ciencia 23, 17-22. file:///C:/Users/User/Downloads/919-3369-2-PB.pdf

Cláudio et al (2012). Efeito de níveis de melão em substituição ao milho moído sobre o desempenho, o consumo e a digestibilidade dos nutrientes em ovinos Morada Nova. R. Bras. Zootec41164-171. https://www.scielo.br/pdf/rbz/v41n1/24.pdf

Eduardo, G. A. (2014). Elaboração alternativa de produtos a partir de resíduos alimentares. file:///C:/Users/User/Downloads/162-856-1-PB.pdf

Fábio, C. da S.; et al (2010). Desarrollo de las pequeñas industrias rurales de la caña de azúcar en Iberoamérica: Melaza, Panela y Azúcar Moreno. Jornadas ibero-americanas sobre optimización energética y ecológica de la agroindústria de la caña de azucar. http://oa.upm.es/6622/1/pequenas.pdf

FEDNA.2020. Melazas de caña. Fundación Española para el Desarrollo de la Nutrición Animal: http://www.fundacionfedna.org/ingredientes_para_pienso s/melazas-de-ca\%C3\%B1a

ICEX. 2020. El mercado de frutas y hortalizas de Brasil. Oficina Económica y Comercial de la Embajada de España en São Paulo. http://www.ivace.es/Internacional_Informes-Publicaciones/Pa\%C3\%ADses/Brasil/Brasilfrutasverdurasiicex2020.pdf

Instituto Adolfo Lutz. Métodos físico-químicos para análise de alimentos. Instituto Adolfo Lutz. 1020p.

Josiane, A. da C. (2018). Da semente a farinha: semeando a sustentabilidade no aproveitamento resíduo do melão Cantalupe (Cucumis melo L. var. reticulatus). Dissertação de Mestrado apresentada ao Programa de Pós-Graduação em Nutrição, da Universidade Federal do Rio Grande Norte, como requisito parcial à obtenção do título de Mestre em Nutrição. https://repositorio.ufrn.br/jspui/bitstream/123456789/26602/1/Sementefarinhasemeando_Cunha_2018.pdf

José, V. B, et al (2007). Uso alternativo de la melaza de la caña de azúcar residual para la síntesis de espumas rígidas de poliuretano (erp) de uso industrial. Tecnol. Ciencia Ed. (IMIQ) http://repositorio.conare.ac.cr/bits tream/handle/20.500.12337/3249/USO\%20ALTERNATIVO\%20DE\%20LA\%20 MELAZA\%20DE\%20LA\%20CA\%C3\%91A_VEGA.pdf?sequence=1

Journal of AOAC INTERNATIONAL. (1922). https://doi.org/10.1093/jaoac/75.1.200

Karen et al. (2011). Evaluación de las características nutricionales y fisicoquímicas del jugo del fruto de Opuntia ficus-indica (TUNA). Trabajo de grado presentado para optar al título de licenciatura en química y farmacia. Universidad de El Salvador departamento de química y farmacia. Disponible en: http://ri.ues.edu.sv/id/eprint/2437/1/TRABAJO_DE_GRADUACION_GRUPO_38-10.pdf

Tautiva, L. A. V. (2017). Evaluación de la obtención de melaza por medio de hidrolisis acida de cascaras de plátano Dominico-Hartón. (Musa ABB Simmonds) a nivel laboratorio. Fundación Universidad América de ingenierías programa de ingeniería química. Disponible en: http://repository.uamerica.edu.co/bitstream/20.500.11839/6046/1/6112815-2017-I-IQ.pdf

Rodríguez, L. M. A., et al (2013). Determinación de fenoles, flavonoides y capacidad antioxidante en melaza, azúcar blanco y moreno en el ingenio Chaparrastique por el método de espectrometría ultravioleta-visible. Trabajo de graduación para optar al grado de licenciatura en química y farmacia. Universidad de El Salvador. http://ri.ues.edu.sv/id/eprint/5311/1/16103410.pdf

Velazquez, E, Tournier, H. A., Mordujovich de Buschiazzo, P.,Saavedra, G, \& Schinella, G. R. (2003). Antioxidant activity of Paraguayan plant extracts. Fitoterapia, 74, 91-97. Vit, P., Soler, C., \& Tomas-Barberan, F. A.

Meda, A, Lamien. C. E, Romito, M, Millogo, J. \& Nacoulma, O. G. (2005). Determination of the total phenolic, flavonoid and proline contents in Burkina Fasan honey, as well as their radical scavenging activity. Food Chemistry, 91, 571-577.

Moro, P. J. (2012). Diseño y construcción de un clarificador de melaza para mejorar los procesos de fermentación alcohólica. Universidad Nacional Agraria de la Selva. Facultad de Industrias Alimentarias. http://repositorio.unas.edu.pe/handle/UNAS/263?show=full

Roriz, R. F. C. (2012). Aproveitamento dos resíduos alimentícios obtidos das centrais de abastecimento do estado de Goiás s/a para alimentação humana. Dissertação apresentada à Coordenação do Programa de Pós-Graduação em Ciência e Tecnologia de Alimentos, da Escola de Agronomia e Engenharia de Alimentos, da Universidade Federal de Goiás, como exigência para obtenção do título de Mestre em Ciência e Tecnologia de Alimentos. https://files.cercomp.ufg.br/weby/up/71/o/Dissertacao_Renata_Fleury.pdf

Tamayo, M. (2002). El proceso de la investigación científica. LIMUSA.

Melo, V. L. de L. (2020). Melão in natura como dieta exclusiva para bovinos: Um estudo de caso. Dissertação apresentada ao Mestrado em Produção Animal do Programa de Pós Graduação em Produção Animal da Universidade Federal Rural do Semi-Árido como requisito para obtenção do título de Mestre em Produção Animal. https://repositorio.ufersa.edu.br/bitstream/prefix/5410/1/VitorLLM_DISSERT.pdf

Wagner, D. N. A. (2008). "Caracterización reológica y térmica de miel de dos variedades de caña”. Trabajo de graduación, modalidad Sistema Tutorial, presentado como requisito previo a la obtención del Título de Ingeniero en Alimentos, otorgado por la Universidad Técnica de Ambato a través de la Facultad de Ciencia e Ingeniería en Alimentos. file:///C:/Users/User/Downloads/silo.tips_aprobacion-del-tutor\%20(1).pdf

Yemn, E. W., \& Willis, A. J. (1954). The estimation of carbohydrate in plant extracts by anthrone. The Biochemical Journal, London, 57:508-14. 